\title{
Boundary integral equations for plane orthotropic bodies and exterior regions
}

\author{
G. Szeidl and J. Dudra \\ University of Miskolc, Department of Mechanics \\ 3515 Miskolc-Egyetemváros, Hungary
}

\begin{abstract}
Assuming linear displacements and constant strains and stresses at infinity, we reformulate the equations of the direct boundary element method for plane problems of elasticity. We consider a body made of orthotropic material. The reformulated equations make it possible to attack plane problems on exterior regions without replacing the region by a bounded one.
\end{abstract}

\section{Introduction}

As is well known a large literature studies plane problems for orthotropic bodies, including for instance $[1,2,3]$, as well as the books $[4,5]$ and the references therein. However, as explained by Schiavone [6], the standard formulation for exterior regions has the disadvantage that it is impossible to prescribe a constant stress state at infinity.

The reason is that an assumption about the far field pattern of the displacements is needed in order to establish an appropriate Betti's formula and to prove uniqueness and existence for the exterior Dirchlet and Neuman problems. Unfortunately, this assumption excludes those problems from the theory for which the displacements are linear while the strains and stresses are constant at infinity.

To make progress on plane problems with such displacements, we note that if the direct formulation reproduces this displacement field, then the resulting strain and stress conditions must also be constant at infinity. Consequently, plane problems for the exterior regions can be attacked without replacing the region by a bounded one. The work in [7] and [8] presents such direct formulations by assuming constant strains and stresses at infinity for an isotropic body. For exterior regions [7] reformulates the classical approach to plane problems. For the same class of problems but in a dual formulation [8] sets up the equations of the direct method in terms of stress functions of order one.

The present paper is an attempt to clarify how the formulation changes if we apply the ideas presented in paper [7] to orthotropic bodies.

\section{Basic Equations in Generalized Plane Strain}

Throughout this paper $x_{1}$ and $x_{2}$ are rectangular Cartesian coordinates, referred to an origin $O$. Greek subscripts are assumed to have the range $(1,2)$, summation over repeated subscripts is implied. The doubly connected exterior region under 
consideration is denoted by $A_{e}$ and is bounded by the contour $\mathcal{L}_{o}$. We stipulate that the contour admits a nonsingular parametrization in terms of its arc length $s$. The outer normal is denoted by $n_{\pi}$. In accordance with the notations introduced, $\delta_{\kappa \lambda}$ is the Kronecker symbol, $\partial_{\alpha}$ stands for the derivatives taken with respect to $x_{\alpha}$ and $\epsilon_{3 \kappa \lambda}$ is the permutation symbol. Assuming plane problems let $u_{\kappa}, e_{\kappa \lambda}$ and $t_{\kappa \lambda}$ be the displacement field and the in plane components of strain and stress, respectively. For orthotropic bodies the material constants are denoted by $s_{11}$, $s_{12}=s_{21}, s_{22}$ and $s_{66}$.

For homogenous and orthotropic material the plane problem of classical elasticity is governed by the kinematic equations

$$
e_{\rho \lambda}=\frac{1}{2}\left(\partial_{\rho} u_{\lambda}+\partial_{\lambda} u_{\rho}\right)
$$

Hook's law

$$
\begin{aligned}
& t_{11}=c_{11} e_{11}+c_{12} e_{22}, \\
& t_{22}=c_{12} e_{11}+c_{22} e_{22}, \\
& t_{12}=t_{21}=2 c_{66} e_{12},
\end{aligned}
$$

where

$$
c_{11}=\frac{s_{22}}{d}, \quad c_{12}=c_{21}=-\frac{s_{12}}{d}, \quad c_{22}=\frac{s_{11}}{d}, \quad c_{66}=\frac{1}{s_{66}}, \quad d=s_{11} s_{22}-s_{12}^{2} ;
$$

and the equilibrium equations

$$
t_{\rho \lambda} \partial_{\lambda}+b_{\rho}=0
$$

which should be complemented with appropriate boundary conditions not detailed here since they play no role in the present investigations. The basic equation for $u_{\lambda}$ takes the form

$$
\mathcal{D}_{\rho \lambda} u_{\lambda}+b_{\rho}=0
$$

where the differential operator $\mathcal{D}_{\rho \lambda}$ has the form

$$
\left[\mathcal{D}_{\rho \lambda}\right]=\left[\begin{array}{cc}
c_{11} \partial_{1}^{2}+c_{66} \partial_{2}^{2} & \left(c_{12}+c_{66}\right) \partial_{1} \partial_{2} \\
\left(c_{21}+c_{66}\right) \partial_{2} \partial_{1} & c_{22} \partial_{2}^{2}+c_{66} \partial_{1}^{2}
\end{array}\right]
$$

Let $Q\left(\xi_{1}, \xi_{2}\right)$ and $M\left(x_{1}, x_{2}\right)$ be two points in the plane (the source point and the field point). We shall assume temporarily that the point $Q$ is fixed. The distance between $Q$ and $M$ is $R$, the position vector of $M$ relative to $Q$ is $r_{\kappa}$. The small circle as a subscript (for instance $M_{\circ}$ or $Q_{\circ}$ ) indicates that the corresponding points, i.e., $Q$ or $M$ are taken on the contour.

It is obvious that

$$
r_{\alpha}(M, Q)=x_{\alpha}(M)-\xi_{\alpha}(Q)=x_{\alpha}-\xi_{\alpha} .
$$


Let us introduce the following notations

$$
\begin{gathered}
\lambda_{1}+\lambda_{2}=\left(2 s_{12}+s_{66}\right) / s_{22}, \\
\lambda_{1} \lambda_{2}=s_{11} / s_{22}, \\
A_{\alpha}=s_{12}-\lambda_{\alpha} s_{22}, \\
\rho_{\alpha}^{2}=\lambda_{\alpha} r_{1}^{2}+r_{2}^{2}, \\
D=\frac{1}{2 \pi\left(\lambda_{1}-\lambda_{2}\right) s_{22}} .
\end{gathered}
$$

For our later considerations we note that equations (7) and (8) imply

$$
\lambda_{1,2}=\frac{2 s_{21}+s_{66}}{2 s_{22}} \pm \sqrt{\left(\frac{2 s_{21}+s_{66}}{2 s_{22}}\right)^{2}-\frac{s_{11}}{s_{22}}} .
$$

The well known singular fundamental solutions for the basic equation $(5 \mathrm{a})[1,3]$ are given by the formulas

$$
\begin{aligned}
& U_{11}(M, Q)=D\left(\sqrt{\lambda_{1}} A_{2}^{2} \ln \rho_{1}-\sqrt{\lambda_{2}} A_{1}^{2} \ln \rho_{2}\right), \\
& U_{12}(M, Q)=D A_{1} A_{2} \arctan \frac{\left(\sqrt{\lambda_{1}}-\sqrt{\lambda_{2}}\right) r_{1} r_{2}}{\sqrt{\lambda_{1}} \sqrt{\lambda_{2}} r_{1}^{2}+r_{2}^{2}}, \\
& U_{21}(M, Q)=U_{12}\left(M_{\circ}, Q\right), \\
& U_{22}(M, Q)=-D\left(\frac{A_{1}^{2} \ln \rho_{1}}{\sqrt{\lambda_{1}}}-\frac{A_{2}^{2} \ln \rho_{2}}{\sqrt{\lambda_{2}}}\right)
\end{aligned}
$$

and

$$
\begin{aligned}
& T_{11}(M, Q)=D\left[\frac{\sqrt{\lambda_{2}} A_{1}}{\rho_{2}^{2}}-\frac{\sqrt{\lambda_{1}} A_{2}}{\rho_{1}^{2}}\right]\left(r_{1} n_{1}+r_{2} n_{2}\right), \\
& T_{12}(M, Q)=D\left\{\left(\frac{\sqrt{\lambda_{1}} A_{1}}{\rho_{1}^{2}}-\frac{\sqrt{\lambda_{2}} A_{2}}{\rho_{2}^{2}}\right) r_{1} n_{2}-\left(\frac{1}{\sqrt{\lambda_{1}}} \frac{A_{1}}{\rho_{1}^{2}}-\frac{1}{\sqrt{\lambda_{2}}} \frac{A_{2}}{\rho_{2}^{2}}\right) r_{2} n_{1}\right\}, \\
& T_{21}(M, Q)=D\left\{\left(\frac{\lambda_{1} \sqrt{\lambda_{1}} A_{2}}{\rho_{1}^{2}}-\frac{\lambda_{2} \sqrt{\lambda_{2}} A_{1}}{\rho_{2}^{2}}\right) r_{1} n_{2}-\left(\frac{\sqrt{\lambda_{1}} A_{2}}{\rho_{1}^{2}}-\frac{\sqrt{\lambda_{2}} A_{1}}{\rho_{2}^{2}}\right) r_{2} n_{1}\right\}, \\
& T_{22}(M, Q)=D\left[\frac{\sqrt{\lambda_{1}} A_{1}}{\rho_{1}^{2}}-\frac{\sqrt{\lambda_{2}} A_{2}}{\rho_{2}^{2}}\right]\left(r_{1} n_{1}+r_{2} n_{2}\right),
\end{aligned}
$$

where

$$
\mathfrak{u}_{\lambda}(M)=U_{\lambda \kappa}(M, Q) e_{\kappa}(Q) \quad \text { and } \quad t_{\lambda}(M)=T_{\lambda \kappa}(M, Q) e_{\kappa}(Q)
$$

are the displacement vector and stress vector on a line element with a normal $n_{\lambda}=n_{\lambda}(M)$ to it at the point $M$ due to the force $e_{\kappa}=e_{\kappa}(Q)$ at $Q$. 


\section{Basic formulas for exterior regions}

Figure 1 depicts a triple connected region $A_{e}^{\prime}$ bounded by the contours $\mathcal{L}_{o}, \mathcal{L}_{\varepsilon}$ and the circle $\mathcal{L}_{R}$ with radius ${ }_{e} R$ and center at $O$. Here $\mathcal{L}_{\varepsilon}$ is the contour of the neighborhood $A_{\varepsilon}$ of $Q$ with radius $R_{\varepsilon}$ while ${ }_{e} R$ is sufficiently large so that the region bounded by $\mathcal{L}_{R}$ covers both $\mathcal{L}_{0}$, and $\mathcal{L}_{\varepsilon}$. If ${ }_{e} R \rightarrow \infty$ and $R_{\varepsilon} \rightarrow 0$ then clearly $A_{e}^{\prime} \rightarrow A_{e}$.

Let $u_{\kappa}(M)$ and $g_{\kappa}(M)$ be sufficiently smooth - continuously differentiable at least twice - but otherwise arbitrary displacement fields on $A_{e}$. The stresses obtained from these displacement fields are denoted by $t_{\lambda \kappa}\left[u_{\rho}(M)\right]$ and $t_{\lambda \kappa}\left[g_{\rho}(M)\right]$ respectively.

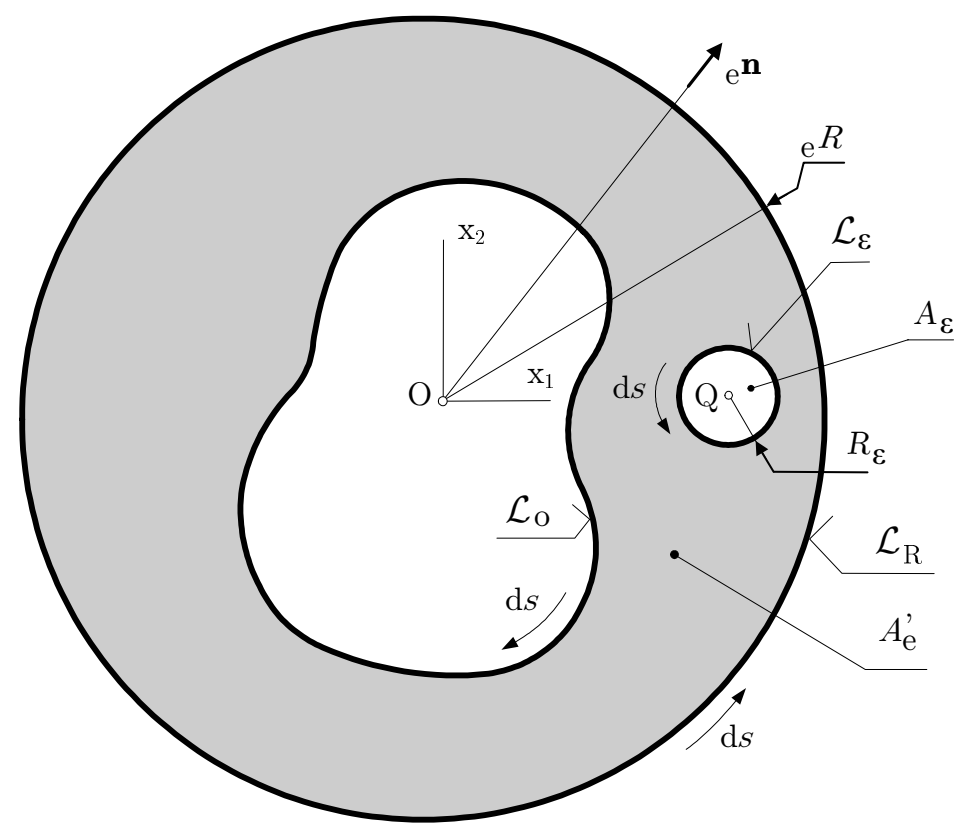

Figure 1:

Equation

$$
\begin{aligned}
& \int_{A_{e}^{\prime}}\left[u_{\lambda}(M)\left(\mu \stackrel{M}{\mathcal{D}}{ }_{\lambda \sigma} g_{\sigma}(M)\right)-g_{\lambda}(M)\left(\mu \stackrel{M}{\mathcal{D}}{ }_{\lambda \sigma} u_{\sigma}(M)\right)\right] \mathrm{d} A_{M}= \\
& \quad=\oint_{\mathcal{L}_{o}}\left[u_{\lambda}\left(M_{\circ}\right) t_{\lambda \kappa}\left[g_{\rho}\left(M_{\circ}\right)\right] n_{\kappa}\left(M_{\circ}\right)-g_{\lambda}\left(M_{\circ}\right) t_{\lambda \kappa}\left[u_{\rho}\left(M_{\circ}\right)\right] n_{\kappa}\left(M_{\circ}\right)\right] \mathrm{d} s_{M_{\circ}} \\
& \quad+\oint_{\mathcal{L}_{\varepsilon}}\left[u_{\lambda}\left(M_{\circ}\right) t_{\lambda \kappa}\left[g_{\rho}\left(M_{\circ}\right)\right] n_{\kappa}\left(M_{\circ}\right)-g_{\lambda}\left(M_{\circ}\right) t_{\lambda \kappa}\left[u_{\rho}\left(M_{\circ}\right)\right] n_{\kappa}\left(M_{\circ}\right)\right] \mathrm{d} s_{M_{\circ}} \\
& +\oint_{\mathcal{L}_{R}}\left[u_{\lambda}\left(M_{\circ}\right) t_{\lambda \kappa}\left[g_{\rho}\left(M_{\circ}\right)\right] n_{\kappa}\left(M_{\circ}\right)-g_{\lambda}\left(M_{\circ}\right) t_{\lambda \kappa}\left[u_{\rho}\left(M_{\circ}\right)\right] n_{\kappa}\left(M_{\circ}\right)\right] \mathrm{d} s_{M_{\circ}},
\end{aligned}
$$


in which $M$ over a letter denotes derivatives taken with respect to the point coordinates $M$ and $n_{\kappa}\left(M_{\circ}\right)$ is the outward normal, is the primal Somigliana identity applied to the triple connected region $A_{e}^{\prime}$.

Let $g_{\lambda}(Q)=U_{\lambda \kappa}(M, Q) e_{\kappa}(Q)$, which is a non singular elastic state of the plane in $A_{e}^{\prime}$. We regard $u_{\lambda}(M)$ as a different elastic state in the region $A_{e}$. Further we assume that $u_{\lambda}(M)$ has the far field pattern (asymptotic behavior)

$$
\tilde{u}_{\kappa}(M)=c_{\kappa}+\varepsilon_{3 \rho \kappa} x_{\rho} \omega+e_{\kappa \beta}(\infty) x_{\beta}
$$

as $x_{\beta}$ or equivalently $M$ tends to infinity. Here $c_{\kappa}$ is a translation, $\omega$ is a rotation in finite, $c_{\kappa}+\varepsilon_{3 \rho \kappa} x_{\rho} \omega$ is the corresponding rigid body motion, $e_{\kappa \beta}(\infty)$ is a constant strain tensor at infinity and $e_{\kappa \beta}(\infty) x_{\beta}$ is the corresponding displacement field.

The stresses induced by the strains $e_{\kappa \beta}(\infty)$ can be obtained by the Hooke law:

$$
\begin{aligned}
& t_{11}(\infty)=c_{11} e_{11}(\infty)+c_{12} e_{22}(\infty), \\
& t_{22}(\infty)=c_{12} e_{11}(\infty)+c_{22} e_{22}(\infty), \\
& t_{12}(\infty)=t_{21}(\infty)=2 c_{66} e_{12}(\infty),
\end{aligned}
$$

Substituting the above quantities into the Somigliana identity, we obtain

$$
\begin{aligned}
\int_{A_{e}^{\prime}}\left[u_{\lambda}(M)\right. & \left.\left(\mu \stackrel{M}{\mathcal{D}_{\lambda \sigma}} U_{\sigma \kappa}(M, Q)\right)-\left(\mu \mathcal{D}_{\lambda \sigma}^{M} u_{\sigma}(M)\right) U_{\lambda \kappa}(M, Q)\right] \mathrm{d} A_{M} e_{\kappa}(Q)= \\
= & \oint_{\mathcal{L}_{o}}\left[u_{\lambda}\left(M_{\circ}\right) T_{\lambda \kappa}\left(M_{\circ}, Q\right)-t_{\lambda}\left(M_{\circ}\right) U_{\lambda \kappa}\left(M_{\circ}, Q\right)\right] \mathrm{d} s_{M_{\circ}} e_{\kappa}(Q)+ \\
& +\oint_{\mathcal{L}_{\varepsilon}}\left[u_{\lambda}\left(M_{\circ}\right) T_{\lambda \kappa}\left(M_{\circ}, Q\right)-t_{\lambda}\left(M_{\circ}\right) U_{\lambda \kappa}\left(M_{\circ}, Q\right)\right] \mathrm{d} s_{M_{\circ}} e_{\kappa}(Q) \\
& +\oint_{\mathcal{L}_{R}}\left[u_{\lambda}\left(M_{\circ}\right) T_{\lambda \kappa}\left(M_{\circ}, Q\right)-t_{\lambda}\left(M_{\circ}\right) U_{\lambda \kappa}\left(M_{\circ}, Q\right)\right] \mathrm{d} s_{M_{\circ}} e_{\kappa}(Q)
\end{aligned}
$$

since $t_{\lambda \kappa}\left[u_{\rho}\left(M_{\circ}\right)\right] n_{\kappa}\left(M_{\circ}\right)=t_{\lambda}\left(M_{\circ}\right)$ is the stress on the contour and obviously

$$
t_{\lambda \kappa}\left[g_{\rho}\left(M_{\circ}\right)\right] n_{\kappa}\left(M_{\circ}\right)=T_{\lambda \kappa}\left(M_{\circ}, Q\right) e_{\kappa}(Q) \text {. }
$$

In the sequel we shall assume that there are no body forces. This assumption has no effect on the result we will obtain.

It is clear that one can omit $e_{\kappa}(Q)$. Regarding the equation obtained by omitting $e_{\kappa}(Q)$, our goal is to compute its limit as $R_{\varepsilon} \longrightarrow 0$ and ${ }_{e} R \longrightarrow \infty$. As is well known, the left hand side vanishes under the conditions detailed above(see [4] ), and hence

$\oint_{\mathcal{L}_{o}} \cdots+\lim _{R_{\varepsilon} \longrightarrow 0} \oint_{\mathcal{L}_{\varepsilon}} \cdots=u_{\kappa}(Q)+\oint_{\mathcal{L}_{o}}\left[u_{\lambda}(\stackrel{o}{M}) T_{\lambda \kappa}\left(M_{\circ}, Q\right)-t_{\lambda}\left(M_{\circ}\right) U_{\lambda \kappa}\left(M_{\circ}, Q\right)\right] \mathrm{d} s_{M_{\circ}}$.

Consequently

$$
\begin{aligned}
u_{\kappa}(Q)=\lim _{e R \longrightarrow \infty} & \oint_{\mathcal{L}_{R}}\left[t_{\lambda}\left(M_{\circ}\right) U_{\lambda \kappa}\left(M_{\circ}, Q\right)-u_{\lambda}\left(M_{\circ}\right) T_{\lambda \kappa}\left(M_{\circ}, Q\right)\right] \mathrm{d} s_{M_{\circ}}+ \\
& +\oint_{\mathcal{L}_{\circ}}\left[t_{\lambda}\left(M_{\circ}\right) U_{\lambda \kappa}\left(M_{\circ}, Q\right)-u_{\lambda}\left(M_{\circ}\right) T_{\lambda \kappa}\left(M_{\circ}, Q\right)\right] \mathrm{d} s_{M_{\circ}} .
\end{aligned}
$$


In order to establish the first Somigliana formula for the exterior region, we need to find the limit of the first integral on the right hand side.

\section{Somigliana formulas modified for exterior regions}

Our goal in this section is to prove that

$$
\begin{aligned}
I_{\kappa}=\lim _{e R \longrightarrow \infty} \oint_{\mathcal{L}_{R}}\left[t_{\lambda}\left(M_{\circ}\right) U_{\lambda \kappa}\left(M_{\circ}, Q\right)-u_{\lambda}\left(M_{\circ}\right) T_{\lambda \kappa}\left(M_{\circ}, Q\right)\right] \mathrm{d} s_{M_{\circ}}= \\
=c_{\kappa}+\varepsilon_{3 \rho \kappa} \xi_{\rho} \omega+e_{\kappa \beta}(\infty) \xi_{\beta}=\tilde{u}_{\kappa}(Q) .
\end{aligned}
$$

The proof is based on the first Somigliana formula for inner regions and requires simple tools only ${ }^{1}$.

Consider the simple connected region $A_{R}$ bounded by the circle $\mathcal{L}_{R}$ with radius ${ }_{e} R$ and center at $O$. Assume that $Q$ is in the interior of the region. The displacement field $\tilde{u}(M)$ is an elastic state of $A_{R}$ with no body forces. The corresponding strain and stress tensors are denoted by $\tilde{e}_{\kappa \lambda}(M)$ and $\tilde{t}_{\kappa \lambda}(M)$, respectively. The stress vector on the boundary is $\tilde{t}_{\kappa}=\tilde{t}_{\kappa \lambda}\left(M_{o}\right) n_{\kappa}\left(M_{o}\right)$. Since $\tilde{u}(M)$ is an elastic state of the orthotropic inner region $A_{R}$, the first Somigliana formula implies

$$
\tilde{u}_{\kappa}(Q)=\oint_{\mathcal{L}_{R}}\left[\tilde{t}_{\lambda}\left(M_{\circ}\right) U_{\lambda \kappa}\left(M_{\circ}, Q\right)-\tilde{u}_{\lambda}\left(M_{\circ}\right) T_{\lambda \kappa}\left(M_{\circ}, Q\right)\right] \mathrm{d} s_{M_{\circ}} .
$$

Now consider the displacement field

$$
\tilde{u}_{\lambda}(M)=c_{\kappa}+\varepsilon_{3 \rho \kappa} x_{\rho} \omega+e_{\kappa \beta}(\infty) x_{\beta}
$$

which describes the asymptotic behavior of $u_{\lambda}(M)$ in the exterior region $A_{e}$. It can be checked that this displacement field is an elastic state of the entire plane, including the region $A_{R}$, for which the stress tensor is constant, i.e., $\tilde{t}_{\kappa \lambda}(M)=$ $\tilde{t}_{\kappa \lambda}(\infty)$. Given an elastic state of the plane, combining equations (21) and (22) yields

$$
\begin{aligned}
\oint_{\mathcal{L}_{R}}\left[\tilde{t}_{\lambda}\left(M_{\circ}\right) U_{\lambda \kappa}\left(M_{\circ}, Q\right)-\tilde{u}_{\lambda}\left(M_{\circ}\right)\right. & \left.T_{\lambda \kappa}\left(M_{\circ}, Q\right)\right] \mathrm{d} s_{M_{\circ}}= \\
& =\tilde{u}_{\lambda}(Q)=c_{\kappa}+\varepsilon_{3 \rho \kappa} \xi_{\rho} \omega+e_{\kappa \beta}(\infty) \xi_{\beta} .
\end{aligned}
$$

Combining this with the limits

$$
\lim _{e R \longrightarrow \infty} u_{\lambda}(M)=\tilde{u}_{\lambda} \quad \text { and } \quad \lim _{e R \longrightarrow \infty} t_{\lambda}(M)=\tilde{t}_{\lambda},
$$

\footnotetext{
${ }^{1}$ We would like to thank one of the referees for suggesting this simple derivation which replaces our more complicated argument.
} 
and equation (23), we find that the limit of the integral in question in equation (20) is

$$
\begin{aligned}
\lim _{e} \oint_{\mathcal{L}_{R}}\left[t_{\lambda}\left(M_{\circ}\right) U_{\lambda \kappa}\left(M_{\circ}, Q\right)-\right. & \left.u_{\lambda}\left(M_{\circ}\right) T_{\lambda \kappa}\left(M_{\circ}, Q\right)\right] \mathrm{d} s_{M_{\circ}}= \\
& =\tilde{u}_{\kappa}(Q)=c_{\kappa}+\varepsilon_{3 \rho \kappa} \xi_{\rho} \omega+e_{\kappa \beta}(\infty) \xi_{\beta} .
\end{aligned}
$$

Consequently, the first and modified Somigliana formulas immediately follow from equations (19) and (20):

$$
\begin{array}{r}
u_{\kappa}(Q)=\tilde{u}_{\kappa}(Q)+\oint_{\mathcal{L}_{\circ}}\left[t_{\lambda}\left(M_{\circ}\right) U_{\lambda \kappa}\left(M_{\circ}, Q\right)-u_{\lambda}\left(M_{\circ}\right) T_{\lambda \kappa}\left(M_{\circ}, Q\right)\right] \mathrm{d} s_{M_{\circ}} \\
Q \in A_{e}
\end{array}
$$

If $Q=Q_{\circ}$ is on $\mathcal{L}_{o}$, our previous logic about the integral taken on $\mathcal{L}_{R}$ is unaffected. Consequently

$$
\begin{aligned}
& C_{\kappa \rho} u_{\rho}\left(Q_{\circ}\right)=\tilde{u}_{\kappa}\left(Q_{\circ}\right)+ \\
& +\oint_{\mathcal{L}_{o}}\left[t_{\lambda}\left(M_{\circ}\right) U_{\lambda \kappa}\left(M_{\circ}, Q_{\circ}\right)-u_{\lambda}\left(M_{\circ}\right) T_{\lambda \kappa}\left(M_{\circ}, Q_{\circ}\right)\right] \mathrm{d} s_{M_{\circ}} \quad Q=Q_{\circ} \in \mathcal{L}_{o}
\end{aligned}
$$

where $C_{\kappa \rho}=\delta_{\kappa \rho} / 2$ if the contour is smooth at $Q_{0}$. This is the integral equation of the direct method, or the second Somigliana formula for exterior regions.

If $Q$ is inside the contour $\mathcal{L}_{o}$, i.e., in region $A_{i}$, then it is easy to show that

$$
\begin{array}{r}
0=e_{\kappa \beta}(\infty) \xi_{\beta}(Q)+\oint_{\mathcal{L}_{\circ}}\left[t_{\lambda}\left(M_{\circ}\right) U_{\lambda \kappa}\left(M_{\circ}, Q\right)-u_{\lambda}\left(M_{\circ}\right) T_{\lambda \kappa}\left(M_{\circ}, Q\right)\right] \mathrm{d} s_{M_{\circ}} \\
Q=Q_{\circ} \in A_{i}
\end{array}
$$

which is the third Somigliana formula for exterior regions.

Using the formulae set up for the strains in Appendix A and the Hooke law (17) we can calculate the stresses:

$$
\begin{aligned}
t_{\alpha \beta}(Q)=t_{\alpha \beta}(\infty)+\oint_{\mathcal{L}_{\circ}} t_{\lambda}\left(M_{\circ}\right) \hat{\mathcal{D}}_{\lambda \alpha \beta}\left(M_{\circ}, Q\right) \mathrm{d} s_{M_{\circ}-} \\
-\oint_{\mathcal{L}_{\circ}} u_{\lambda}\left(M_{\circ}\right) \mathcal{S}_{\lambda \alpha \beta}\left(M_{\circ}, Q\right) \mathrm{d} s_{M_{\circ}} \quad Q \in A_{e}
\end{aligned}
$$

where

$$
\begin{array}{ll}
\hat{\mathcal{D}}_{\lambda 11}=c_{11} \mathcal{D}_{\lambda 11}+c_{12} \mathcal{D}_{\lambda 22}, & \hat{\mathcal{S}}_{\lambda 11}=c_{11} \mathcal{S}_{\lambda 11}+c_{12} \mathcal{S}_{\lambda 22}, \\
\hat{\mathcal{D}}_{\lambda 11}=c_{12} \mathcal{D}_{\lambda 11}+c_{22} \mathcal{D}_{\lambda 22}, & \hat{\mathcal{S}}_{\lambda 11}=c_{12} \mathcal{S}_{\lambda 11}+c_{22} \mathcal{S}_{\lambda 22}, \\
\hat{\mathcal{D}}_{\lambda 12}=2 c_{66} \mathcal{D}_{\lambda 12}=\hat{\mathcal{D}}_{\lambda 21}, & \hat{\mathcal{S}}_{\lambda 12}=2 c_{66} \mathcal{S}_{\lambda 12}=\hat{\mathcal{S}}_{\lambda 21}
\end{array}
$$




\section{Behavior at infinity}

Our goal in this section is to compute the limit of representation (26) as $Q \rightarrow \infty$. This will lead to a characterization of the asymptotic behavior of $u_{\kappa}(Q)$. If this behavior is the same what we have assumed, i.e., if the limit coincides with (16), then we confirm that the results of the previous section are correct. It is clear from representation (26) that it is sufficient to show that the following relations hold

$$
\begin{aligned}
& \lim _{Q\left(\xi_{1}, \xi_{2}\right) \longrightarrow \infty} \oint_{\mathcal{L}_{o}} t_{\lambda}\left(M_{\circ}\right) U_{\lambda \kappa}\left(M_{\circ}, Q\right) \mathrm{d} s_{M_{\circ}}=0, \\
& \lim _{Q\left(\xi_{1}, \xi_{2}\right) \longrightarrow \infty} \oint_{\mathcal{L}_{o}} u_{\lambda}\left(M_{\circ}\right) T_{\lambda \kappa}\left(M_{\circ}, Q\right) \mathrm{d} s_{M_{\circ}}=0 .
\end{aligned}
$$

In order to find the limit of the above integrals we have to set up asymptotic relations for the fundamental solutions $U_{\lambda \kappa}\left(M_{\circ}, Q\right)$ and $T_{\lambda \kappa}\left(M_{\circ}, Q\right)$ if $Q \longrightarrow \infty$.

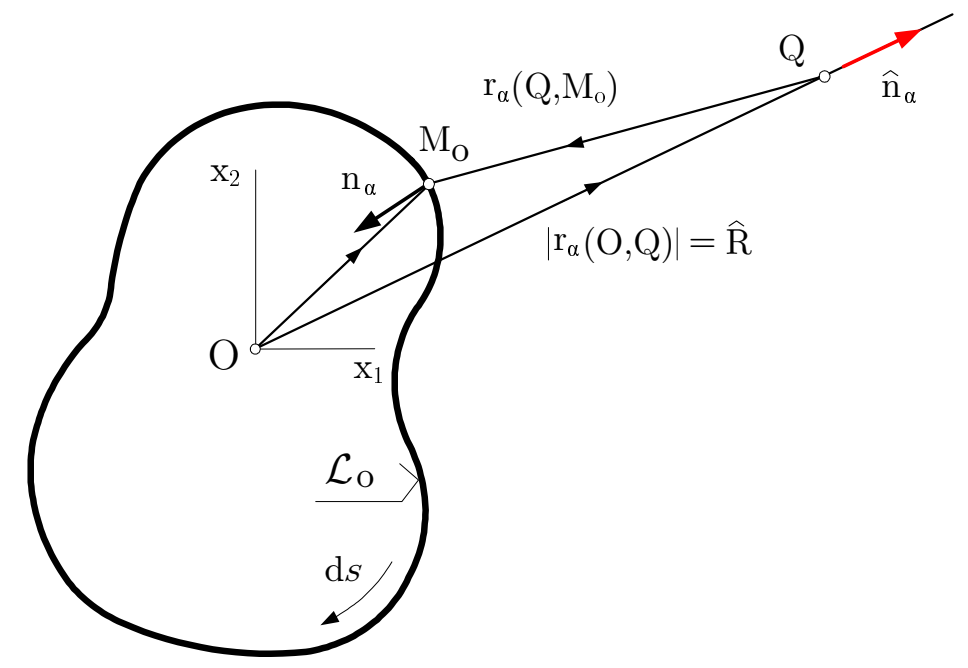

Figure 2:

Using the notations introduced in Figure 2. as well as equations (6) and (10) we have

$$
\begin{gathered}
r_{\alpha}(\stackrel{o}{M}, Q)=x_{\alpha}(\stackrel{o}{M})-\xi_{\alpha}(Q)=x_{\alpha}-\xi_{\alpha}=-\hat{R}\left(\hat{n}_{\alpha}-\frac{x_{\alpha}}{\hat{R}}\right) \approx-\hat{R} \hat{n}_{\alpha}\left|\hat{n}_{\alpha}\right|=1 \\
\rho_{\alpha}=\sqrt{\lambda_{\alpha} r_{1}^{2}+r_{2}^{2}} \approx \hat{R} \sqrt{\lambda_{\alpha} \hat{n}_{1}^{2}+\hat{n}_{2}^{2}} \\
\ln \rho_{\alpha} \approx \ln \hat{R}+\frac{1}{2} \ln \left(\lambda_{\alpha} \hat{n}_{1}^{2}+\hat{n}_{2}^{2}\right) .
\end{gathered}
$$


Substituting equations (32a,b,c) into (13) then performing some manipulations, we obtain the following asymptotic relations for the fundamental solution of order one:

$$
\begin{gathered}
U_{11}\left(M_{\circ}, Q\right) \approx D\left[\sqrt{\lambda_{1}} A_{2}^{2}-\sqrt{\lambda_{2}} A_{1}^{2}\right] \ln \hat{R}, \\
U_{12}\left(M_{\circ}, Q\right)=U_{21}\left(M_{\circ}, Q\right) \approx D A_{1} A_{2} \arctan \frac{\left(\sqrt{\lambda_{1}}-\sqrt{\lambda_{2}}\right) \hat{n}_{1} \hat{n}_{2}}{\sqrt{\lambda_{1}} \sqrt{\lambda_{2}} \hat{n}_{1}^{2}+\hat{n}_{2}^{2}}, \\
U_{22}\left(M_{\circ}, Q\right) \approx-D\left[\frac{A_{1}^{2}}{\sqrt{\lambda_{1}}}-\frac{A_{2}^{2}}{\sqrt{\lambda_{2}}}\right] \ln \hat{R} .
\end{gathered}
$$

It is obvious that asymptotically $U_{\lambda \kappa}\left(M_{\circ}, Q\right) \approx U_{\lambda \kappa}(Q)$, i.e., the kernel in integral $(31)_{1}$ is independent of $M_{\circ}$.

Consequently

$$
\begin{aligned}
\lim _{Q\left(\xi_{1}, \xi_{2}\right) \longrightarrow \infty} \oint_{\mathcal{L}_{o}} t_{\lambda}\left(M_{\circ}\right) U_{\lambda \kappa}\left(M_{\circ}, Q\right) \mathrm{d} s_{M_{\circ}} & = \\
& =\lim _{Q\left(\xi_{1}, \xi_{2}\right) \longrightarrow \infty} U_{\lambda \kappa}(Q) \underbrace{\oint_{\mathcal{L}_{o}} t_{\lambda}\left(M_{\circ}\right) \mathrm{d} s_{M_{\circ}}}_{\text {resultant }}=0 .
\end{aligned}
$$

By repeating the line of thought leading to the asymptotic relations $(33 \mathrm{a}, \mathrm{b}, \mathrm{c})$, for the fundamental solutions of order two we obtain

$$
\begin{gathered}
T_{11}\left(M_{\circ}, Q\right) \approx-\frac{D}{\hat{R}}\left(\frac{\sqrt{\lambda_{2}} A_{1}}{\lambda_{2} \hat{n}_{1}^{2}+\hat{n}_{2}^{2}}-\frac{\sqrt{\lambda_{1}} A_{2}}{\lambda_{1} \hat{n}_{1}^{2}+\hat{n}_{2}^{2}}\right)=\frac{\tilde{T}_{11}(Q)}{\hat{R}}, \\
T_{12}\left(M_{\circ}, Q\right) \approx-\frac{D}{\hat{R}}\left[\frac{1-\lambda_{2}}{\sqrt{\lambda_{2}}} \frac{A_{2}}{\lambda_{2} \hat{n}_{1}^{2}+\hat{n}_{2}^{2}}-\frac{1-\lambda_{1}}{\sqrt{\lambda_{1}}} \frac{A_{1}}{\lambda_{1} \hat{n}_{1}^{2}+\hat{n}_{2}^{2}}\right] \hat{n}_{1} \hat{n}_{2}=\frac{\tilde{T}_{12}(Q)}{\hat{R}}, \\
T_{21}\left(M_{\circ}, Q\right) \approx-\frac{D}{\hat{R}}\left[\frac{\sqrt{\lambda_{1}}\left(\lambda_{1}-1\right) A_{2}}{\lambda_{1} \hat{n}_{1}^{2}+\hat{n}_{2}^{2}}-\frac{\sqrt{\lambda_{2}}\left(\lambda_{2}-1\right) A_{1}}{\lambda_{2} \hat{n}_{1}^{2}+\hat{n}_{2}^{2}}\right] \hat{n}_{1} \hat{n}_{2}=\frac{\tilde{T}_{21}(Q)}{\hat{R}}, \\
T_{22}\left(M_{\circ}, Q\right) \approx-\frac{D}{\hat{R}}\left(\frac{\sqrt{\lambda_{2}} A_{1}}{\lambda_{1} \hat{n}_{1}^{2}+\hat{n}_{2}^{2}}-\frac{\sqrt{\lambda_{1}} A_{2}}{\lambda_{2} \hat{n}_{1}^{2}+\hat{n}_{2}^{2}}\right)=\frac{\tilde{T}_{22}(Q)}{\hat{R}},
\end{gathered}
$$

where $\tilde{T}_{\lambda \kappa}=\tilde{T}_{\lambda \kappa}(Q)$ is defined by the above equations. Since for large $\hat{R}$ the kernel $T_{\lambda \kappa}\left(M_{\circ}, Q\right)$ is independent of $M_{\circ}$ and tends to zero, it follows that

$$
\begin{aligned}
\lim _{Q\left(\xi_{1}, \xi_{2}\right) \longrightarrow \infty} \oint_{\mathcal{L}_{o}} u_{\lambda}\left(M_{\circ}\right) T_{\lambda \kappa}\left(M_{\circ}, Q\right) \mathrm{d} s_{M_{\circ}} & = \\
= & \lim _{Q\left(\xi_{1}, \xi_{2}\right) \longrightarrow \infty} \frac{\tilde{T}_{\lambda \kappa}(Q)}{\hat{R}} \oint_{\mathcal{L}_{o}} u_{\lambda}\left(M_{\circ}\right) \mathrm{d} s_{M_{\circ}}=0 .
\end{aligned}
$$

This verifies that the asymptotic behavior of the displacement representation (26) is as expected. 


\section{$6 \quad$ Examples}

In this section we solve two external boundary value problems. First we consider the plane with a circular hole (Figure 3.b.); second the plane with a rigid inclusion (Figure 3.c.). The material is birch for which $s_{11}=8.497 \times 10^{-5}, s_{12}=s_{21}=$ $-6.11 \times 10^{-2}, s_{22}=1.6999 \times 10^{-4}$ and $s_{66}=1.456 \times 10^{-3}$. We assume that $\tau_{12}(\infty)=\sigma_{22}(\infty)=0$ and $\sigma_{11}(\infty)=p=$ constant. For completeness Figure 3.a. shows the region to use if we solve the integral equation of the direct method in its traditional form, i.e., if the exterior region is replaced by a bounded one.

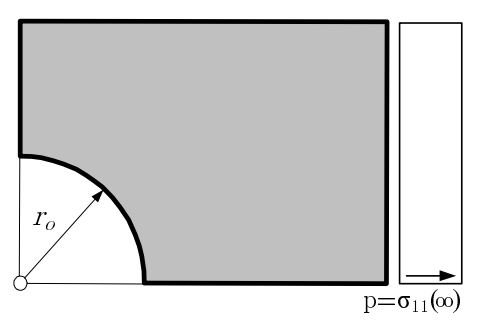

(a)
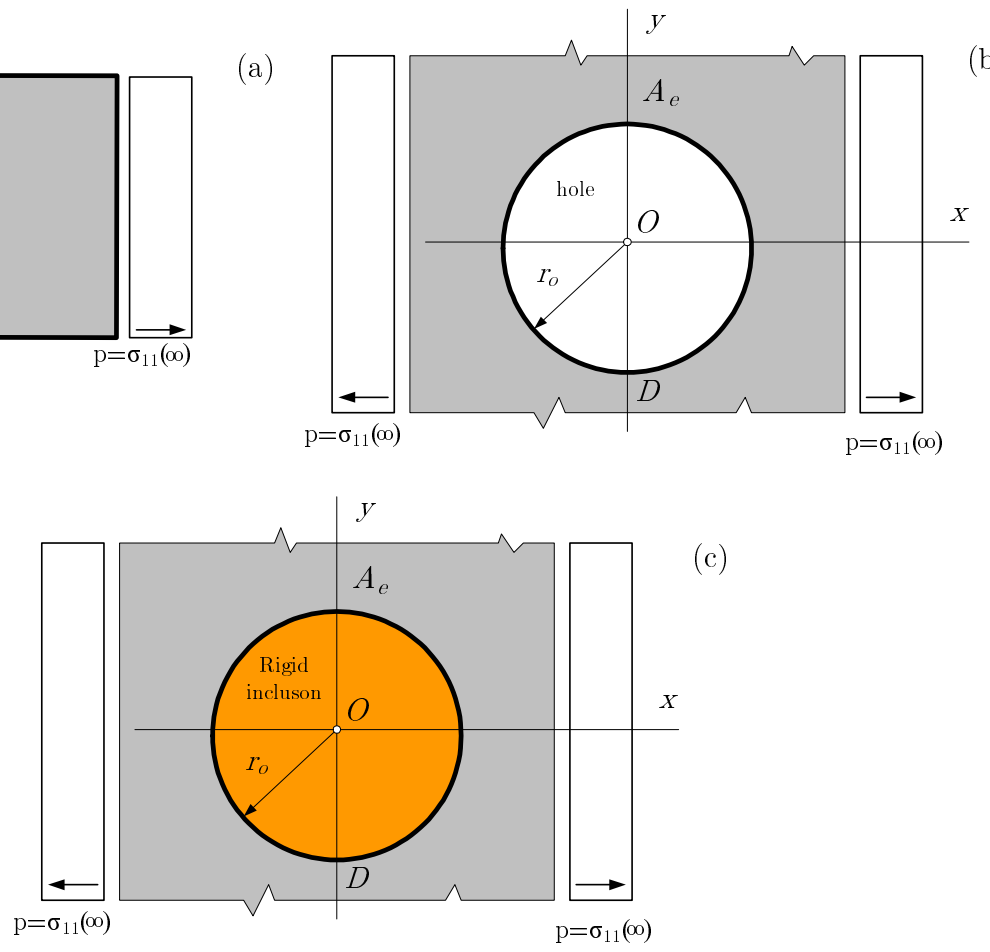

(c)

Figure 3:

Lekhtniski's book [9] contains closed form solutions for the stresses on the boundary, as well as numerical values which can be found in Table 17. on page 197. In this paper we show the results as computed by solving integral equation (27) (typeset in red) and the results taken from [9] - see Tables 1. and 2. We used a polar coordinate system, and the tables contain the quotients $\sigma_{\theta} / p$ for the plane with circular hole and $\sigma_{r} / p \tau_{r \theta} / p, \sigma_{\theta} / p$ for the plane with the circular inclusion. 


\begin{tabular}{|c|rr|}
\hline \multirow{2}{*}{ Polar angle } & \multicolumn{2}{|c|}{ Circular hole } \\
\cline { 2 - 3 } & \multicolumn{1}{|c|}{$\sigma_{\theta} / p$} & {$[9]$, p.197 } \\
\hline $0^{\circ}$ & -0.7071 & -0.707 \\
$15^{\circ}$ & -0.3399 & -0.340 \\
$30^{\circ}$ & 0.0692 & 0.069 \\
$45^{\circ}$ & 0.4040 & 0.404 \\
$60^{\circ}$ & 0.9644 & 0.966 \\
$75^{\circ}$ & 2.5771 & 2.577 \\
$90^{\circ}$ & 5.4514 & 5.453 \\
\hline
\end{tabular}

Table 1: Results for the circular hole

\begin{tabular}{|c|cr|rr|rr|}
\hline \multirow{2}{*}{ Polar angle } & \multicolumn{7}{|c|}{ Rigid kernel } \\
\cline { 2 - 7 } & $\sigma_{r} / p$ & {$[9], \mathrm{p} .197$} & \multicolumn{1}{rr}{$/ p$} & {$[9], \mathrm{p} .197$} & $\sigma_{\theta} / p$ & {$[9], \mathrm{p} .197$} \\
\hline $0^{\circ}$ & 1.2363 & 1.237 & 0.0000 & 0.000 & 0.0445 & 0.044 \\
$15^{\circ}$ & 1.1562 & 1.156 & -0.2994 & -0.299 & 0.0934 & 0.093 \\
$30^{\circ}$ & 0.9370 & 0.937 & -0.5185 & -0.519 & 0.2697 & 0.270 \\
$45^{\circ}$ & 0.6378 & 0.698 & -0.5987 & -0.599 & 0.5154 & 0.516 \\
$60^{\circ}$ & 0.3383 & 0.388 & -0.5185 & -0.519 & 0.6989 & 0.699 \\
$75^{\circ}$ & 0.1192 & 0.119 & -0.2994 & -0.299 & 0.5637 & 0.564 \\
$90^{\circ}$ & 0.0389 & 0.039 & 0.0000 & 0.000 & 0.0028 & 0.003 \\
\hline
\end{tabular}

Table 2: Results for the rigid inclusion

We used partially discontinuous elements of order two. The results obtained are in good agreement with those taken from Lekhtniski's book [9].

\section{Concluding remarks}

For the sake of completeness, note that [10] by Constanda gives an asymptotic expansion for the displacements at infinity which ensures the validity of the Betti formula for exterior regions. Under this condition the total strain energy stored in the region is bounded. In addition uniqueness and existence proofs are easy to give.

We have modified the Somigliana formulas for exterior regions by assuming that the strains are constants and accordingly the displacements are linear at infinity. Under this condition the strain energy density is bounded (but not necessarily the strain energy), and there is no need to replace the exterior region by a finite one if a constant stress condition is prescribed at infinity. This can be an advantage if one considers an infinite plane with holes or cracks in it and the plane is subjected to constant stresses at infinity and an attempt is made to determine the stresses in finite. It is easy to modify existing codes to perform computations. Results for two simple problem are also presented. 


\section{Acknowledgment}

The support provided by the Hungarian National Research Foundation within the framework of the project OTKA T046834 is gratefully acknowledged.

\section{References}

1. Rizzo, R. J. and Shippy, D. J., A method for stress determination in plane anisotropic elastic bodies, J. Composite Materials, vol. 4, pp. 36-61, 1970.

2. Vable, M. and Sikarskie, D. L., Stress analysis in plane orthotropic material by the boundary element method, Int. J. Solids Structures, vol. 24(1), pp. 1-11, 1988.

3. Huang, L., Sun, X., Liu, Y., and Cen, Z., Parameter identification for twodimensional orthotropic material bodies by the boundary element method, Engineering Analysis with Boundary Elements, vol. 28(2), pp. 109-121, 2004.

4. Banarjee, P. K. and Butterfield, R., Boundary Element Methods in Engineering Science, Mir, Moscow, 1984.

5. Banarjee, P. K., The Bondary Element Methods in Engineering, McGraw-Hill, New York, 1994.

6. Schiavone, P. and Ru, C.-Q., On the exterior mixed problem in plane elasticity, Mathematics and Mechanics of Solids, vol. 1, pp. 335-342, 1996.

7. Szeidl, G., Boundary integral equations for plane problems - remark to the formulation for exterior regions, Publications of the University of Miskolc, Series D, Natural Sciences, Mathematics, vol. 40(1), pp. 79-88, 1999.

8. Szeidl, G., Boundary integral equations for plane problems in terms of stress functions of order one, Journal of Computational and Applied Mechanics, vol. 2(2), pp. 237-261, 2001.

9. Lekhnitski, S. G., Theory of Elasticity of an Anisotropic Body, Nauka, Moscow, 1977, the second and revised Russian edition.

10. Constanda, C., The Boundary Iintegral Equation Method in Plane Elasticity, Proceedings of the American Mathematical Society, vol. 123(11), pp. 33853396, 1995 . 


\section{A Formulae for strains}

Making use of equations (13), (14) and (26) from the kinematic equations (1) we obtain

$$
e_{\alpha \beta}=\oint_{\mathcal{L}_{o}} t_{\lambda}\left(M_{\circ}\right) \mathcal{D}_{\lambda \alpha \beta}\left(M_{\circ}, Q\right) \mathrm{d} s_{M_{\circ}}-\oint_{\mathcal{L}_{o}} u_{\lambda}\left(M_{\circ}\right) \mathcal{S}_{\lambda \alpha \beta}\left(M_{\circ}, Q\right) \mathrm{d} s_{M_{\circ}} \quad Q \in A_{e}
$$

where

$$
\begin{array}{ll}
\mathcal{D}_{\lambda 11}=U_{\lambda 1} \partial_{1}, & \mathcal{S}_{\lambda 11}=T_{\lambda 1} \partial_{1}, \\
\mathcal{D}_{\lambda 12}=\frac{1}{2}\left(U_{\lambda 2} \partial_{1}+U_{\lambda 1} \partial_{2}\right)=\mathcal{D}_{\lambda 21}, & \mathcal{S}_{\lambda 12}=\frac{1}{2}\left(T_{\lambda 2} \partial_{1}+T_{\lambda 1} \partial_{2}\right)=\mathcal{D}_{\lambda 21}, \\
\mathcal{D}_{\lambda 22}=U_{\lambda 2} \partial_{2}, & \mathcal{S}_{\lambda 22}=T_{\lambda 2} \partial_{2} .
\end{array}
$$

If we introduce the notations

$$
\begin{aligned}
& b_{1}=\frac{\sqrt{\lambda_{1}}}{\rho_{1}^{2}}, \quad b_{2}=\frac{\sqrt{\lambda_{2}}}{\rho_{2}^{2}}, \quad c=\sqrt{\lambda_{1}} \sqrt{\lambda_{2}} r_{1}^{2}+r_{2}^{2}, \quad d=\left(\sqrt{\lambda_{1}}-\sqrt{\lambda_{2}}\right) r_{2}, \\
& k_{1}=\frac{\lambda_{1}^{\frac{3}{2}} A_{1}}{\rho_{1}^{2}}, \quad k_{2}=\frac{\lambda_{2}^{\frac{3}{2}} A_{2}}{\rho_{2}^{2}}, \quad f_{1}=\frac{A_{1}}{\left(\lambda_{1}^{\frac{3}{2}} r_{1}^{2}+r_{2}^{2}\right)^{2}}, \quad f_{2}=\frac{A_{2}}{\left(\lambda_{2}^{\frac{3}{2}} r_{1}^{2}+r_{2}^{2}\right)^{2}}
\end{aligned}
$$

for the derivatives in equation (38) we have

$$
\begin{aligned}
& \partial_{1} U_{11}=D\left(b_{2} \lambda_{2} A_{1}^{2} r_{1}-b_{1} \lambda_{1} A_{2}^{2} r_{1}\right), \\
& \partial_{1} U_{12}=\frac{D A_{1} A_{2}}{1+d^{2} r_{1}^{2}}\left(2 \sqrt{\lambda_{1}} \sqrt{\lambda_{2}} d r_{1}^{2}-c d\right)=\partial_{1} U_{21}, \\
& \partial_{1} U_{22}=D\left(b_{1} A_{1}^{2} r_{1}-b_{2} A_{2}^{2} r_{1}\right), \\
& \partial_{2} U_{11}=D\left(b_{2} A_{1}^{2} r_{2}-b_{1} A_{2}^{2} r_{2}\right), \\
& \partial_{2} U_{12}=\frac{D A_{1} A_{2}}{1+d^{2} r_{1}^{2}}\left[2 d r_{1} r_{2}-c\left(\sqrt{\lambda_{1}}-\sqrt{\lambda_{2}}\right) r_{1}\right]=\partial_{2} U_{21}, \\
& \partial_{2} U_{22}=-D\left(\frac{A_{2}^{2} r_{2}}{\rho_{2}^{2} \sqrt{\lambda_{2}}}-\frac{A_{1}^{2} r_{2}}{\rho_{1}^{2} \sqrt{\lambda_{1}}}\right)
\end{aligned}
$$

and

$$
\begin{aligned}
\partial_{1} T_{11} & =D\left[\frac{2 b_{2} \lambda_{2} A_{1} r_{1}}{\rho_{2}^{2}}-\frac{2 b_{1} \lambda_{1} A_{2} r_{1}}{\rho_{1}^{2}}\right]\left(r_{1} n_{1}+r_{2} n_{2}\right)-D\left[b_{2} A_{1}-b_{1} A_{2}\right] n_{1}, \\
\partial_{1} T_{12} & =D\left[\frac{2 b_{1} \lambda_{1} A_{1} r_{1}}{\rho_{1}^{2}}-\frac{2 b_{2} \lambda_{2} A_{2} r_{1}}{\rho_{2}^{2}}\right] r_{1} n_{2}-D\left[b_{1} A_{1}-b_{2} A_{2}\right] n_{2}- \\
& -D\left[2 f_{1} \lambda_{1} r_{1}-2 f_{2} \lambda_{2} r_{1}\right] r_{2} n_{1} \\
\partial_{1} T_{21} & =D\left[\frac{2 b_{1} \lambda_{1}^{2} A_{2} r_{1}}{\rho_{1}^{2}}-\frac{2 b_{1} \lambda_{2}^{2} A_{1} r_{1}}{\rho_{2}^{2}}\right] r_{1} n_{2}-D\left[b_{1} \lambda_{1} A_{2}-b_{2} \lambda_{2} A_{1}\right] n_{2}- \\
& -D\left[\frac{2 b_{1} \lambda_{1} A_{2} r_{1}}{\rho_{1}^{2}}-\frac{2 b_{2} \lambda_{2} A_{1} r_{1}}{\rho_{2}^{2}}\right] r_{2} n_{1}, \\
\partial_{1} T_{22} & =D\left[\frac{2 b_{1} \lambda_{1} A_{1} r_{1}}{\rho_{1}^{2}}-\frac{2 b_{2} \lambda_{2} A_{2} r_{1}}{\rho_{2}^{2}}\right]\left(r_{1} n_{1}+r_{2} n_{2}\right)-D\left[b_{1} A_{1}-b_{2} A_{2}\right] n_{1},
\end{aligned}
$$




$$
\begin{aligned}
\partial_{2} T_{11} & =D\left[\frac{b_{2} A_{1} 2 r_{2}}{\rho_{2}^{2}}-\frac{b_{1} A_{2} 2 r_{2}}{\rho_{1}^{2}}\right]\left(r_{1} n_{1}+r_{2} n_{2}\right)-D\left[b_{2} A_{1}-b_{1} A_{2}\right] n_{2}, \\
\partial_{2} T_{12} & =D\left[\frac{b_{1} A_{1} 2 r_{2}}{\rho_{1}^{2}}-\frac{b_{2} A_{2} 2 r_{2}}{\rho_{2}^{2}}\right] r_{1} n_{2}-D\left[2 f_{1} r_{2}-2 f_{2} r_{2}\right] r_{2} n_{1}+ \\
& +D\left[f_{1}-f_{2}\right] n_{1}, \\
\partial_{2} T_{21} & =D\left[\frac{b_{1} \lambda_{1} A_{2} 2 r_{2}}{\rho_{1}^{2}}-\frac{b_{2} \lambda_{2} A_{1} 2 r_{2}}{\rho_{2}^{2}}\right] r_{1} n_{2}-D\left[\frac{b_{1} A_{2} 2 r_{2}}{\rho_{1}^{2}}-\frac{b_{2} A_{1} 2 r_{2}}{\rho_{2}^{2}}\right] r_{2} n_{1}+ \\
& +D\left[b_{1} A_{2}-b_{2} A_{1}\right] n_{1}, \\
\partial_{2} T_{22} & =D\left[\frac{b_{1} A_{1} 2 r_{2}}{\rho_{1}^{2}}-\frac{b_{2} A_{2} 2 r_{2}}{\rho_{2}^{2}}\right]\left(r_{1} n_{1}+r_{2} n_{2}\right)-D\left[b_{1} A_{1}-b_{2} A_{2}\right] n_{2} .
\end{aligned}
$$

